\title{
On matching conditions for cosmological perturbations
}

\author{
Nathalie Deruelle \\ Département d'Astrophysique Relativiste et de Cosmologie \\ Centre National de la Recherche Scientifique, \\ Observatoire de Paris, 92195 Meudon Cedex, France, and \\ Department of Applied Mathematics and Theoretical Physics \\ University of Cambridge, Silver Street Cambridge CB3 9EW, England \\ V.F. Mukhanov \\ Institut für Theoretishe Physik, \\ ETH, Hönggerberg, CH-8001 Zürich, Switzerland, and, \\ Département d'Astrophysique Relativiste et de Cosmologie \\ Centre National de la Recherche Scientifique, \\ Observatoire de Paris, 92195 Meudon Cedex, France
}

(September 3, 2018)

\begin{abstract}
We derive the matching conditions for cosmological perturbations in a Friedmann Universe where the equation of state undergoes a sharp jump, for instance as a result of a phase transition. The physics of the transition which is needed to follow the fate of the perturbations is clarified. We dissipate misleading statements made recently in the literature [1] concerning the predictions of the primordial fluctuations from inflation and confirm standard results. Applications to string cosmology are considered.
\end{abstract}




\section{INTRODUCTION}

The evolution of metric perturbations in a Friedmann Universe is well known (see, e.g. [2] and refs therein). They can be classified into scalar perturbations, which couple to the matter density inhomogeneities, and freely propagating gravitational waves (we shall ignore here the vectorial perturbations). In inflationary models [3] the perturbations of both types are produced from primordial quantum fluctuations, and their spectra after inflation are nearly scale invariant $[4,5]$. However the amplitudes of the gravitational waves and scalar perturbations are significantly different at all scales after the transition from the inflationary to the matter dominated stage.

Let us, for instance consider a Universe which evolves from a phase (-) with the scale factor $a_{-} \propto t^{p_{-}}$to a phase $(+)$such that $a_{+} \propto t^{p_{+}}$. If the transition from the $(-)$and to the $(+)$ phase happens at time $t_{t}$, then the ratio of the amplitude $h^{+}$of the long wavelength gravity waves some time after the transition $\left(t \gg t_{t}\right)$ and directly before it $h^{-}\left(\right.$at $\left.t=t_{t}-0\right)$ is:

$$
\frac{h^{+}}{h_{-}} \rightarrow O(1)
$$

while for the gravitational potential $\Phi$, which characterizes scalar metric perturbations, the result is

$$
\frac{\Phi^{+}}{\Phi^{-}} \rightarrow \frac{1+p_{-}}{1+p_{+}}
$$

We see that for a strongly inflating $(-)$ phase $\left(p_{-} \gg 1\right)$ the amplification of density perturbations can be very big whereas gravity waves are not amplified.

This standard result has however been challenged recently in [1].

We think that there is nothing strange about this difference in the amplification of the gravity waves and density perturbations. Of course, eq. (1.2) doesn't imply that the gravitational potential jumps at the moment of transition. What happens is that the gravitational potential $\Phi$ which can be small immediately before and after the transition is distributed at 
$t=t_{t}+0$ into two modes, both very large in amplitude, one which decays, the other yielding (1.2) (the derivative $\dot{\Phi}$ of the gravitational potential on the other hand can strongly jump at $t_{t}$ in distinction from the gravity waves for which $\dot{h}$ is continuous). As we will show the result depends on the physics of the transition.

In order to make this statement quantitative we derive the matching conditions for cosmological perturbations in models with sharp transitions. The results will be applied to the simplified model of inflation considered in [1] which uses a sharp jump of the equation of state at the end of inflation instead of evolving it rapidly but smoothly as in e.g. [2] (see also e.g. [6. [6]). We think that the results obtained in [1] imply the strongly scale dependent spectrum for scalar perturbation in the relevant for COBE scales. We do not agree with the author of paper [1] who concluded from his consideration that the spectrum is flat taking the parameter $\gamma_{1}$ to be equal 2 on p. 7166, while it should be taken $\gamma_{1} \ll 1$. It will be shown in Sect. $\mathrm{VB}$ that the root for the misleading result [1] lies in inappropriate matching conditions which have no physical justification. We on the other hand shall make the matching on hypersurfaces of constant energy for physical reasons and clarify and confirm the standard results (1.1)-(1.2).

The considerations in this paper actually go beyond clarifying this point. They can be applied to study the evolution of cosmological perturbations in models where the transition from one stage of evolution to an other is not completely specified. We clarify the physical features of the phase transition which are of importance for the evolution of cosmological perturbations. As a particular example we consider the fate of the growing mode in string cosmology after transition from superinflation to the Friedmann era (see Sect. Đ).

The paper is organized as follows. In Section $\mathbb{1}$ we recall the standard results. In Section $[1]$ the general matching conditions in models with sharp transitions are derived. We also discuss there the choice of the hypersurface of transition. In Section $\mathbb{V}$ we write the matching conditions in two particular coordinate systems: the longitudinal and the synchronous reference frame used in [1]. Finally, in Section $\nabla$, we apply our results to concrete examples. Conclusions are drawn in Section V1. 


\section{BACKGROUND AND PERTURBATIONS}

Consider a quasi-isotropic spatially flat Friedmann Universe with metric

$$
d s^{2}=a^{2}\left\{(1+2 \phi) d \eta^{2}-2 B_{, i} d x^{i} d \eta-\left[(1-2 \psi) \delta_{i k}+2 E_{, i k}+h_{i k}\right] d x^{i} d x^{k}\right\}
$$

where $a(\eta)$ is the scale factor, a comma means the derivative with respect to spatial coordinates and the four functions $\phi, \psi, B$ and $E$ characterize the metric perturbations of scalar

type. The tensor $h_{i k}$, which is taken transverse and traceless $\left(h_{, k}^{i k}=h_{i}^{i}=0\right)$ corresponds to gravity waves (all spatial indices will be raised with $\delta^{i k}$ ). We ignore here the vector perturbations. Under coordinate transformations $\eta \rightarrow \tilde{\eta}=\eta+\xi^{0}, x^{i} \rightarrow \tilde{x}^{i}=x^{i}+\xi^{, i}$, the scalar metric perturbations transform as:

$$
\begin{aligned}
\phi \rightarrow \tilde{\phi} & =\phi-\mathcal{H} \xi^{0}-\xi^{0^{\prime}} \quad ; \quad B \rightarrow \tilde{B}=B+\xi^{0}-\xi^{0^{\prime}} ; \\
\psi \rightarrow \tilde{\psi}=\psi-\mathcal{H} \xi^{0} \quad ; & E \rightarrow \tilde{E}=E-\xi ;
\end{aligned}
$$

where $\mathcal{H}=a^{\prime} / a=H a$ and a prime denotes a derivative with respect to conformal time $\eta$.

From (2.2) we see that two simple gauge invariant quantities, characterizing the scalar perturbations, can be built, e.g. [8,1]

$$
\Phi=\phi+\frac{1}{a}\left[\left(B-E^{\prime}\right) a\right]^{\prime}, \Psi=\psi-\mathcal{H}\left(B-E^{\prime}\right) .
$$

In the longitudinal gauge $\left(B_{\ell}=E_{\ell}=0\right)$ these gauge invariant variables coincide with the metric perturbations. Therefore the calculations in this coordinate system are completely the same as those in terms of the gauge invariant variables $\Phi$ and $\Psi$. As for the gravity waves they are gauge invariant: $\tilde{h}_{i j}=h_{i j}$.

If we consider the perturbations with scales much bigger than the Hubble radius $H^{-1}$, then the solution of Einstein equations in the long wavelength approximation can be written down as (see e.g. [2]):

$$
h=D_{g}+S_{g} \int \frac{d t}{a^{3}}
$$

for gravity waves and 


$$
\Phi=\Psi=S_{s} \frac{H}{a}+D_{s}\left(\frac{1}{a} \int a d t\right)
$$

for scalar perturbations in the case of matter an adiabatic perfect fluid or a scalar field. Here a dot denotes a derivative with respect to cosmic time $t=\int a d \eta$, and $H=\dot{a} / a$. The constants of integration $D, S$ are fixed by physical considerations, e.g. by imposing that the perturbations be in their quantum ground state when they are inside the Hubble radius during an inflationary era. We note that the solution (2.5) can be obtained if we integrate the equation for the quantity (see, for instance [2,5, [, 9, [10])

$$
\zeta=\Phi+\frac{\mathcal{H}}{\mathcal{H}^{2}-\mathcal{H}^{\prime}}\left(\Phi^{\prime}+\mathcal{H} \Phi\right)
$$

which is "conserved" in the long wavelength approximation.

Let us pause onto the model described in the Introduction, where the scale factor evolves as $a \propto t^{p_{-}}$before the transition and as $a \propto t^{p_{+}}$after it. Then using eqs. (2.4), (2.5) one gets that $h^{-} \rightarrow D_{g}, \Phi^{-} \rightarrow D_{s} /\left(1+p_{-}\right)$and after the transition after the subdominant mode has decayed: $h^{+} \rightarrow D_{g}, \Phi^{+} \rightarrow D_{s} /\left(1+p_{+}\right)$. From here immediately follow the results (1.1), (1.2). Since during inflation $1+p_{-} \propto H^{2} / \dot{H} \gg 1$ we see that the scalar perturbations can be very strongly amplified in the course of the transition.

The standard derivation described above was criticized in [11] on the grounds that the long wavelength approximation used breaks down for scalar perturbations of cosmologically relevant scales at the moment of the transition. It is true that the condition of applicability of the long wavelength approximation for scalar perturbations can be significantly different from simply imposing that the wavelength be bigger than the Hubble scale (see [2] and [7]). Therefore we believe that it is quite useful to clarify how the perturbations evolve in models with a sharp change of the equation of state and discuss the features of the physics of the transition which are relevant for cosmological perturbations. 


\section{MATCHING CONDITIONS}

Suppose that the stress-energy tensor, which governs the evolution of the metric (2.1) via Einstein's equations undergoes a finite discontinuity on a spacelike hypersurface $\Sigma$ determined by the equation $q\left(\eta, x^{i}\right)=$ const., where $q$ is 4 -scalar. In a slightly inhomogeneous Universe it can be decomposed into a "homogeneous" part $q_{0}(\eta)$ plus a small inhomogeneity $\delta q\left(\eta, x^{i}\right)$, that is $q=q_{0}+\delta q$. Under coordinate transformations $\eta=\tilde{\eta}=\eta+\xi^{0}$ etc..., $\delta q$ changes as

$$
\delta q \rightarrow \widetilde{\delta} q=\delta q-q_{0}^{\prime} \xi^{0}
$$

We shall consider below specific examples for the function $q$. The hypersurface $\Sigma$ divides the manifold into two regions, $(-)$ and $(+)$ which should be matched in such a way that the induced 3-metric on $\Sigma$ and its extrinsic curvature be continuous (in the absence of surface layers) [12]. The simplest way to do the calculation is to go to the "tilde coordinate system": $\eta \rightarrow \tilde{\eta}, x^{i} \rightarrow \tilde{x}^{i}$, where the equation for $\Sigma$ becomes $\tilde{\eta}=$ const.. It means that in this coordinate system $\widetilde{\delta q}=0$ and eq. (3.1) determines the transformation:

$$
\xi^{0}=\frac{\delta q}{q_{0}^{\prime}} \quad, \quad \xi-\text { arbitrary }
$$

From this, one first immediately gets that for the background and gravity waves the matching conditions impose that the scale factor $a, h_{i k}$ and their first time derivatives be continuous on $\Sigma$ in any coordinate system. For the scalar perturbations we can easily calculate the extrinsic curvature $\widetilde{\delta K}_{j}^{i}$ in the "tilde coordinate system" and the matching conditions for them read:

$$
\begin{aligned}
& {[\tilde{\psi}]_{ \pm}=[\tilde{E}]_{ \pm}=0} \\
& {\left[\widetilde{\delta K}_{j}^{i}\right]_{ \pm}=-\frac{1}{a}\left[\delta_{j}^{i}\left(\mathcal{H} \tilde{\phi}+\tilde{\psi}^{\prime}\right)+\left(\tilde{B}-\tilde{E}^{\prime}{ }^{\prime}{ }_{, j}{ }_{, j}\right]_{ \pm}=0\right.}
\end{aligned}
$$

where $[\tilde{\psi}]_{ \pm} \equiv \tilde{\psi}_{+}-\tilde{\psi}_{-}$etc. From eq. $\left.3.3 \mathrm{~b}\right)$ it follows that $\left(\mathcal{H} \tilde{\phi}+\tilde{\psi}^{\prime}\right)$ and $\left(\tilde{B}-\tilde{E}^{\prime}\right)$ should be separatly continuous. Expressing $\tilde{\psi}, \tilde{B}$ etc... in terms of $\psi, B$ etc... in the original 
coordinate system with the help of (2.2) where $\xi^{0}$ should be taken from (3.2), we finally get the matching conditions on $\Sigma: q_{0}+\delta q=$ const. in an arbitrary coordinate system:

$$
\begin{aligned}
& {\left[\psi+\mathcal{H} \delta q / q_{0}^{\prime}\right]_{ \pm}=0,} \\
& {[E-\xi]_{ \pm}=0,} \\
& {\left[B-E^{\prime}+\delta q / q_{0}^{\prime}\right]_{ \pm}=0,} \\
& {\left[\mathcal{H} \phi+\psi^{\prime}+\left(\mathcal{H}^{\prime}-\mathcal{H}^{2}\right) \delta q / q_{0}^{\prime}\right]_{ \pm}=0 .}
\end{aligned}
$$

Eq. (3.4b) is empty since $\xi$ can always be chosen so that it is satisfied.

Now it is time to specify $\Sigma$, that is the scalar $q$. Suppose matter is an adiabatic perfect fluid with stress-energy tensor $T_{\nu}^{\mu}=(p+\varepsilon) u^{\mu} u_{\nu}-p \delta_{\nu}^{\mu}$, and that the equation of state $p=p(\varepsilon)$ undergoes a sudden change at some moment of time. Then it is clear that on $\Sigma$ the energy density $\varepsilon$ should be constant (that is, $\Sigma: q \equiv \varepsilon_{0}+\delta \varepsilon=$ const.), since the pressure depends only on $\varepsilon$. The background energy density $\varepsilon_{0}$ and its perturbations $\delta \varepsilon$ can be expressed in terms of the scale factor $a$, and the metric perturbations $\phi, \psi$ etc... via the linearized $0-i$ Einstein eqs. and thus the eqs. (3.4) can be written down entirely in terms of the metric perturbations. We will do it in next Section for various gauges.

If matter is a scalar field $\varphi$ with stress-energy tensor $T_{\nu}^{\mu}=\varphi^{, \mu} \varphi_{, \nu}-\delta_{\nu}^{\mu}\left(\frac{1}{2} \varphi^{, \lambda} \varphi_{, \lambda}-V(\varphi)\right)$ which suddenly decays into ultrarelativistic particles then $\Sigma$ should be taken to be the hypersurface $\varepsilon=\frac{1}{2} \varphi^{, \lambda} \varphi_{, \lambda}+V(\varphi)=$ const., where $\varepsilon$ is the "effective" energy of a scalar field considered as a "perfect fluid" with 4 -velocitie $u_{\mu}=\varphi_{, \mu} /\left(\varphi^{, \lambda} \varphi_{, \lambda}\right)^{1 / 2}$.

\section{LONGITUDINAL VS. SYNCHRONOUS GAUGE}

\section{A. Longitudinal gauge}

The longitudinal gauge conditions: $E_{\ell}=B_{\ell}=0$, define a unique coordinate system. In

this coordinate system the matching conditions (2.4) on the hypersurface $\Sigma: \varepsilon_{0}+\delta \varepsilon_{\ell}=$ const simplify to: 


$$
\begin{aligned}
& {\left[\frac{\delta \varepsilon_{\ell}}{\varepsilon_{0}^{\prime}}\right]_{ \pm}=0, \quad\left[\psi_{\ell}\right]_{ \pm}=0,} \\
& {\left[\mathcal{H} \phi_{\ell}+\psi_{\ell}^{\prime}+\mathcal{H}^{\prime} \delta \varepsilon_{\ell} / \varepsilon_{0}\right]_{ \pm}=0 .}
\end{aligned}
$$

Now we will use the background $0-0$ Einstein equation to express $\varepsilon_{0}$ and $\varepsilon_{0}^{\prime}$ in terms of the scale factor and its derivatives:

$$
\varepsilon_{0}=\frac{3 \mathcal{H}^{2}}{\kappa a^{2}}, \quad \varepsilon_{0}^{\prime}=\frac{6}{\kappa} \frac{\mathcal{H}}{a^{2}}\left(\mathcal{H}^{\prime}-\mathcal{H}^{2}\right)
$$

where $\kappa=8 \pi G$, and the $i-k\left(i \neq k\right.$ ) (if is supposed that $\delta T_{k}^{i}=0$ for $i \neq k$ together with 0 - 0 linearized Einstein equations in the longitudinal gauge (see Sec. in [2])

$$
\psi_{\ell}=\phi_{\ell}, \quad \delta \varepsilon_{\ell}=\frac{6}{\kappa a^{2}}\left[\frac{1}{3} \Delta \phi_{\ell}-\mathcal{H}\left(\mathcal{H} \phi_{\ell}+\phi_{\ell}^{\prime}\right)\right] .
$$

Then substituting these eqs. in (4.2) we arrive at the following two independent matching conditions (the third one becomes redundant):

$$
\begin{aligned}
& {\left[\phi_{\ell}\right]_{ \pm}=0} \\
& {\left[\zeta_{\ell}+\frac{1}{3} \frac{\Delta \phi_{\ell}}{\mathcal{H}^{\prime}-\mathcal{H}^{2}}\right]_{ \pm}=0}
\end{aligned}
$$

where $\zeta_{\ell}$ is the "conserved" quantity defined by (2.6) (recall that $\Phi=\phi_{\ell}$ ). The second term proportional to $\Delta \phi_{\ell}$ in $(4.5 \mathrm{~b})$ can be neglected for the perturbations with scales bigger than the Hubble scale $H^{-1}$. Then we see that for these long wavelength perturbations the matching condition (4.5b) reduces to the widely used conservation law for $\zeta_{\ell}$. It is worth to mention that for long wavelength perturbations the continuity of $\phi_{\ell}$ and $\zeta_{\ell}$ also implies that the 3-velocity $u_{\ell}^{i}$ should be continous on $\Sigma$.

We conclude this paragraph by noting that all Eqs. (3.1)-(3.4) can be turned into relations between gauge invariant quantities by changing $\psi_{\ell}$, into $\Psi$, $\phi_{\ell}$ into $\Phi$ etc. This remark allows to readily translate these Eqs. in any other reference system.

\section{B. Synchronous gauge.}

The synchronous frames are defined by imposing the gauge conditions: $\phi_{s}=B_{s}=0$. It is well known that these conditions do not fix completely the coordinate system. Indeed, 
under the coordinate transformations

$$
\eta_{s} \rightarrow \tilde{\eta}_{s}=\eta+\frac{\lambda}{a}, \quad x^{i} \rightarrow \tilde{x}^{i}=x^{i}+\lambda^{, i} \int \frac{d \eta}{a}
$$

where $\lambda$ is an arbitrary function of the spatial coordinates, the gauge remains synchronous $\left(\tilde{\phi}_{s}=\tilde{B}_{s}=0\right)$ and (see eqs. (2.2)).

$$
\psi_{s} \rightarrow \tilde{\psi}_{s}=\psi_{s}+\frac{\mathcal{H} \lambda}{a} ; E_{s} \rightarrow \tilde{E}_{s}=E_{s}-\lambda \int \frac{d \eta}{a}
$$

The function $\lambda$ and the constant of integration in (4.7) correspond to the so-called fictitious modes which do not lead to any physical inhomogeneities. The contraint eqs. (4.4) in synchronous frames go into (see, e.g., [2]):

$$
\psi_{s}=-E_{s}^{\prime \prime}-2 \mathcal{H} E_{s}^{\prime}, \quad \delta \varepsilon_{s}=\frac{6}{\kappa a^{2}}\left[\frac{1}{3} \Delta\left(\psi_{s}+\mathcal{H} E_{s}^{\prime}\right)-\mathcal{H} \psi_{s}^{\prime}\right]
$$

with the help of eqs. (4.3), (4.8) we can easily deduce from (3.4) the independent matching conditions in synchronous coordinate systems on the hypersurface $\Sigma: \varepsilon_{0}+\delta \varepsilon_{s}=$ const,

$$
\left[\psi_{s}+\mathcal{H} E_{s}^{\prime}\right]_{ \pm}=0, \quad\left[\mathcal{H} E_{s}^{\prime}-\frac{1}{\mathcal{H}^{\prime}-\mathcal{H}^{2}}\left(\frac{1}{3} \Delta\left(\psi_{s}+\mathcal{H} E_{s}^{\prime}\right)-\mathcal{H} \psi_{s}^{\prime}\right)\right]_{ \pm}=0
$$

First we note that the particular combinations of the metric variables entering (4.9) do not depend on the fictitious modes and we can work directly with (4.9) to find the amplitudes of the physical modes without specifying a particular synchronous frame. Second, if we substitute the expressions for $\psi_{\ell}, E_{\ell}$ in terms of $\phi_{\ell}$ in (4.9) we immediatly arrive to (4.5). So the matching conditions (4.9) are completely equivalent to (4.5) as it should be.

We see from (4.9) that, contrarily to what is claimed in [1] (begining of [V], p. 7164), the matching conditions in a general synchronous frame can not be reduced in general to the continuity of metric and its first time derivatives. The reason beeing that the hypersurface $\Sigma$ that we choose on physical grounds as the surface $\varepsilon_{0}+\delta \varepsilon_{s}=$ const is not necessarily the surface $\eta=$ const. However, using the remaining coordinate freedom (4.6) we can pick up the particular synchronous frame in which the hypersurface $\Sigma: \varepsilon_{0}+\delta \varepsilon_{s}=$ const. coincide with the hypersurface $\eta_{s}=$ const. It is easy to see from (4.8), (4.9) that this is achieved if we take $\left.\delta \varepsilon_{s}\right|_{\Sigma}=0$, or in explicit form 


$$
\left.\left(\frac{1}{3} \Delta\left(\psi_{s}+\mathcal{H} E_{s}^{\prime}\right)-\mathcal{H} \psi_{s}^{\prime}\right)\right|_{\Sigma}=0
$$

Then in the particular synchronous frame defined by (4.10) the matching conditions (4.9) reduce to the continuity of $\psi_{s}, \psi_{s}^{\prime}, E_{s}^{\prime}$. The quantity $E_{s}$ itself can always be made continuous on $\Sigma$ since there is an extra constant of integration in synchronous frames. These continuity conditions together with (4.10) completely fix all the constants of the problem. For the long wavelength perturbations when the term $\Delta\left(\psi_{s}+\mathcal{H} E_{s}^{\prime}\right)$ can be ignored, eq. (4.10) simplifies to $\left.\psi_{s}^{\prime}\right|_{\Sigma}=0$. Since the perturbations of the 3-velocity $\delta u_{s}^{i}$ are proportional to $\psi_{s}^{\prime}$ (it follows from $0-i$ Einstein eqs), we conclude that for long wavelength perturbations the coordinate system which satisfies (4.10) is the particular synchronous frame which is comoving on the hypersurface $\Sigma$, that is $\left.\delta u_{s}^{i}\right|_{\Sigma}=0$. Finally, we would like to stress that the matching conditions discussed here for longwave perturbations automatically imply the conservation of $\zeta$ during the transition.

\section{EXAMPLES}

\section{A. From a pure de Sitter to a dust dominated era}

To start with we would like to consider some artificial example to demonstrate that we can generate physical inhomogeneities as a result a of transition even if we start from a pure de Sitter Universe where it is known that the scalar metric perturbation are exactly equal to zero. As we will show the result is determined by the physics of the transition. First we recall some useful formulae which relate the metric perturbations in a synhronous frame to the gauge invariant gravitational potential which can be easily obtained by integrating (2.3) if we use there the conditions $B=\phi=0$ and take into account the fact that $\Psi=\Phi$ :

$$
\psi_{s}=\Phi+\frac{\mathcal{H}}{a} \int \Phi a d \eta, \quad E_{s}=-\int \frac{d \eta}{a} \int \Phi a d \eta
$$

In a pure de Sitter Universe $\Phi=0$. The constant of integration $\lambda$ in (5.1) (for instance, $\left.\psi_{s}=\lambda \frac{\mathcal{H}}{a}\right)$ corresponds to fictitious perturbations. If we fix it to be $\lambda_{0} \neq 0$ then we pick 
up (up to trivial time independent transformations) a particular synchronous coordinate system from the wide class of synchronous flames which are related one to each other by the transformations (4.6). Let us assume (without any physical justification) that the transition from the de Sitter to the dust era happens along the hypersurface $\Sigma: \eta_{0}=$ const. in this particular reference frame characterized by $\lambda_{0}$. The solution for the gravitational potential in a dust dominated universe $\left(a_{m} \propto \eta_{m}^{2}\right)$ is $(\operatorname{see}(2.5))$

$$
\Phi=D_{m}+\frac{S_{m}}{\eta_{m}^{5}}
$$

where both constants of integration $D_{m}$ and $S_{m}$ correspond to physical (nonfictitious) modes. Then using (5.1) one can easily derive the solutions for the metric perturbations in the synchronous frame during the de Sitter stage:

$$
\psi_{s}=\lambda_{0} H_{d . s}, \quad E_{s}^{\prime}=-\frac{\lambda_{0}}{a}
$$

where the Hubble constant $H_{d . s}=\frac{\mathcal{H}}{a}$ does not depend on time in a pure de Sitter Universe, and correspondingly in the dust dominated era:

$$
\psi_{s}=\frac{5}{3} D_{m}+\frac{2 F_{m}}{\eta_{m}^{3}}, E_{s}^{\prime}=-\frac{D_{m}}{3} \eta_{m}+\frac{1}{2} \frac{S_{m}}{\eta_{m}^{4}}-\frac{F_{m}}{\eta_{m}^{2}},
$$

where $F_{m}$ is the constant of integration corresponding to the fictitious mode. From the continuity conditions for $\psi_{s}, \psi_{s}^{\prime}$ and $E_{s}^{\prime}$ which imply the continuity of the metric and extrinsic curvature if $\Sigma: \eta=$ const. (the continuity of $E_{s}$ fixes only the extra constant of integration in (5.2)) one immediatly gets that

$$
D_{m}=\frac{3}{5} \lambda_{0} H_{d . s}, \quad\left(\frac{S_{m}}{\eta_{m}^{5}}\right)_{\Sigma+0}=-\frac{3}{5} \lambda_{0} H_{d . s}, \quad F_{m}=0 .
$$

Since $\mathcal{D}_{m}$ corresponds to physical inhomogeneities we see that as a result of the transition from a pure de Sitter to a matter dominated Universe one produces real scalar perturbations. Of course immediatly after de Sitter stage the gravitational potential is still zero: $\Phi_{\Sigma+0}=0$, but it consists of two modes, one which decays, while the other survives. The final result for the amplitude of the physical perturbations is determined by the hypersurface of decay of the de Sitter Universe (by $\lambda_{0}$ in example considered here). 
Now, in a pure de Sitter space there is nothing which could help us to fix the hypersurface of transition to a matter dominated era. For instance, any hypersurface is an hypersurface of constant energy. So the physical result is uncertain. Therefore the small deviations from a pure de Sitter stage play a crucial role in predicting the resulting inhomogeneities, since they actually define the physics of the transition. The most natural possibility (in the absence of further concrete model) is to take as $\Sigma$ the hypersurface of constant energy density as we did.

\section{B. From inflation to a radiation and then dust dominated era}

Now we consider the model studied in [国]. Namely, we assume that the Universe went through three stages: at first there was inflation $(i)$ with scale factor $a_{i} \propto\left(-\eta_{i}\right)^{-1-\gamma}$, where $0 \ll \gamma \ll 1$, then a radiation dominated era $(r)$ with $a_{r} \propto \eta_{r}$ and finally a dust matter dominated stage $(m): a_{m} \propto \eta_{m}$. Let us denote the times of transition from $i$ to $r$ stage as $\eta_{1}$ and correspondingly from $r$ to $m$ stage as $\eta_{2}$ in terms of conformal time $\eta_{r}$ in the $r$-stage. Under the above parametrization of the scale factors, conformal time $\eta$ jumps during the transitions and to relate, for instance $\eta_{i}$ to $\eta_{r}$ at this point we can use the continuity condition for $\mathcal{H}$. The time $\eta$ can be easily made to be continuous by shifting $\eta_{r}$ and $\eta_{m}$ by constant factors. However it has no impact on the final results and to simplify the formulae we prefer to use the parametrization above. Also from the very beginning we restrict ourselves only to inhomogeneities with scales bigger than the Hubble radius at $\eta=\eta_{2}$, that is $\left(k \eta_{2}\right) \ll 1$, where $k$ - is the comoving wavenumber of planewave perturbations. Then for such perturbations we can write the solutions for the gravitational potential $\Phi$ in the long wavelength approximation during the inflation and radiation dominated stages as:

$$
\begin{aligned}
& \Phi_{i} \simeq \sqrt{\gamma} H_{i}^{k} \\
& \Phi_{r} \simeq D_{r}\left(1-\frac{\left(\omega \eta_{r}\right)^{2}}{10}\right)+\frac{S_{r}}{\eta_{r}^{3}} .
\end{aligned}
$$


We used Planck units and $H_{i}^{k}$ is the value of Hubble constant at the moment of horizon crossing by the perturbation with the wavenumber $k$ during inflation. Imposing the perturbation to be in their quantum grond state during inflation fixes the amplitude in (5.6a) [4] (there is no disagreement in the literature on this point) and we ignored the decaying mode which will be completely irrelevant at the end of inflation. In the radiation stage we keep in $(5.6 \mathrm{~b})$ the first correction $\sim(\omega \eta)^{2}$, where $\omega=k / \sqrt{3}$, to the constant nondecaying mode since at leading order this mode has a vanishing derivative.

The solution (5.6b) can be obtained from well known exact solution [2,1] in the $r$-stage, if we expand it in powers of $\omega \eta$. Using (5.6) one easily gets with the help of (5.1) the following expression for $\psi_{s}$ and $E_{s}^{\prime}$ in a synchronous frame:

$$
\begin{aligned}
\psi_{i} & =\frac{1+2 \gamma}{\sqrt{\gamma}} H_{i}^{k}+(1+\gamma) F_{i}\left(-\eta_{i}\right)^{\gamma}, \\
E_{i}^{\prime} & =-\frac{1}{\sqrt{\gamma}} H_{i}^{k}\left(-\eta_{i}\right)-F_{i}\left(-\eta_{i}\right)^{1+\gamma},
\end{aligned}
$$

in the inflationary stage $(i)$ and

$$
\begin{aligned}
& \psi_{r}=\frac{3}{2}\left(1-\frac{1}{12}\left(\omega \eta_{r}\right)^{2}\right) D_{r}+\frac{F_{r}}{\eta_{r}^{2}} \\
& E_{r}^{\prime}=-\frac{1}{2}\left(1-\frac{1}{20}\left(\omega \eta_{r}\right)^{2}\right) \eta_{r} D_{r}+\frac{S_{r}}{\eta_{r}^{2}}-\frac{F_{r}}{\eta_{r}}
\end{aligned}
$$

in the $r$-stage, where the constants of integration $F_{i}, F_{r}$ correspond to fictitious modes.

The potentials in a dust matter dominated Universe were given before (see formulae (5.2), (5.4). Now if we claim that $\psi, \psi^{\prime}, E^{\prime}$ are continuous two on the transition hypersurfaces, $\eta_{r}=\eta_{1}$ and $\eta_{r}=\eta_{2}$, then we get 6 eqs. for seven unknown constants $D_{r}, S_{r}, D_{m}, S_{m}, F_{i}$, $F_{r}, F_{m}$. Clearly we need one extra condition. The one imposed in [1] is that in the dust dominated era the synchronous coordinate system be also the comoving one. This fixes the constant $F_{m}$ to be zero. Then a straightforward calculation gives us the following result for the constant $D_{m}$ :

$$
D_{m}=\frac{3}{5} \frac{(1+2 \gamma)}{\sqrt{\gamma}}\left(1+\frac{2+\gamma}{12 \gamma}\left(\omega \eta_{m}\right)^{2}\left(\eta_{2} / \eta_{1}\right)^{2}\right)^{-1}
$$

which agrees with [1]]. 
Recall that $D_{m}$ is the gravitational potential in the $m$-stage. Since $\gamma \ll 1$ we see from (5.9) that for cosmologically relevant scales for which $\frac{1}{\gamma}\left(\omega \eta_{2}\right)^{2}\left(\eta_{2} / \eta_{1}\right)^{2} \gg 1$, the spectrum (5.9) is by no means scale invariant (for which $D_{m}$ doesn't depend on $k$ ). We do not agree with the author of paper [1] who concluded on the base of consideration similar to the above one that it implies a scale invariant spectrum in relevant for COBE scales. The reason for disagreement is that he took, as we think mistakenly, in formula for $\mathcal{D}$ on the page 7166 [1] the parameter $\gamma_{1}$ to be equal 2 (it corresponds to $\gamma=-2$ in our notations) instead of $\gamma_{1} \ll 1$. Since the spectrum (5.9) doesn't resemble at all the standard result (see [2]) it is quite interesting to find out the reason for this desagreement.

As we argued in Sect. III, the physics of the transition tells us that it should happen on the hypersurface of constant energy. This implies that, for instance, in an arbitrary synchronous coordinate system the matching conditions should be (4.9). They are equivalent to the continuity conditions for $\psi_{s}, \psi_{s}^{\prime}, E_{s}^{\prime}$ only in the particular synchronous frame which for long wavelength perturbations is close to the comoving one at the moment of transition.

The precise condition which fixed this coordinate system and, accordingly, the amplitudes of the fictitious modes $F$ is given by (4.10). If we decide the synchronous frame to be comoving one in the $m$-stage $\left(F_{m}=0\right)$ then this coordinate system satisfies the requirement (4.10) with very good accuracy for $k \eta_{2} \ll 1$ on the hypersurface of transition from radiation to matter dominated era. The function $F_{r}$ which specifies this particular synchronous frame II in the $r$-stage is ${ }^{\text {II }} F_{r} \sim \omega^{2}\left(\eta_{2}\right)^{4} D_{r}$. However, the frame II is very far from the comoving one on the hypersurface of the first transition from the $i$ to the $r$ stage. Actually in the frame I which is comoving on the hypersurface of the first transition the amplitude of the fictitious mode is ${ }^{\mathrm{I}} F \sim \omega^{2}\left(\eta_{1}\right){ }^{4} D_{r}$. Since ${ }^{\mathrm{II}} F /{ }^{I} F \sim \eta_{2} / \eta_{1} \gg 1$, we see that the frames I and II are very different. As one can check this difference is quite important for the perturbations with $\left(\omega \eta_{2}\right)^{2}\left(\eta_{2} / \eta_{1}\right)^{2} \gg 1$. How then should we proceed to get the correct answer for the final amplitude of scalar perturbations? The simplest way is just to use the matching conditions (4.9) which do not involve the fictitious modes $\propto F$ and permit us immediately to write 4 eqs. for 4 unkonwn constants $D_{r}, S_{r}, D_{m}, S_{m}$. However, if we want to insist on the 
continuity conditions for $\psi_{s}, \psi_{s}^{\prime}$ and $E_{s}^{\prime}$ then we can do it but, after the matching in the particular frame I at $\eta_{r}=\eta_{1}$ we should go to the other synchronous frame II to make the matching at $\eta_{r}=\eta_{2}$. This procedure fixes all the constants of the problem. As one can easily check both ways lead to the standard result for the final amplitude of scalar perturbations:

$$
D_{m}=\frac{3}{5} \frac{H_{i}^{k}}{\sqrt{\gamma}},
$$

for $\gamma \ll 1$, which is very different from (5.9), while for longwave gravity waves $h \simeq H_{i}^{k}$.

Finally there is noting strange about the fact that the final amplitude of scalar perturbations can go to infinity when $\gamma \rightarrow 0$ despite the fact that perturbations during inflation go to zero (see (5.6a)). As it was shown in Sect. $\nabla A$ as a result of the decay of a pure de Sitter stage we can get whatever we want unless the physics of transition is specified. In the model considered above the hypersurface of transition becomes more "inhomogeneous" (we mean its extrinsic curvature) when we approach a pure de Sitter space. The formulae (5.10 has however a limited range of validity since it was obtained in the linear perturbation theory. Thus we can believe it only if $\gamma \gg H_{I}^{2}(\Phi \sim D \ll 1)$. We hope that the above consideration clearly demonstrates that the calculations which are very straightforward in terms of gauge invariant variables (or in the longitudinal gauge) can become sophisticated in synchronous frames.

\section{Fate of the "decaying" mode (applications to string cosmology)}

Finally we want to consider some consequences of the obtained results for string cosmology. It is well established (see for instance, [13]) that in the models with a dilatonic field in the absence of nonperturbative potential there is a stage in the evolution of the Universe which is described by "superinflationary" expansion in the "Brans-Dicke frame". It is usually assumed that this stage should serve the same purposes as ordinary inflation [13]. In the "Einstein frame" this superinflationary stage corresponds to a contracting Universe and the "decaying" mode in (2.5), 


$$
\Phi=S \frac{H}{a}
$$

(we consider only long wavelength inhomogeneities) grows in this contracting Universe [14]. There is therefore the danger that this mode will eventually turn in to a nondecaying mode and create big inhomogeneities in the final Friedmann Universe thus completly invalidating the original model. It was found in [14] that the mode (5.11) has peculiar properties which give us the hope to avoid this danger. However, the question of what will happen with it after the transition from the superinflationary to the Friedmann stage was not clarified. The problem is that there are no realistic models which solve the "graceful exit" problem and allow us to go smoothly from the superinflationary to Friedmann era [15]. In the absence of such a model we will assume that the transition happens as a result of a change in the effective equation of state along a hypersurface of constant energy. On the basis of the results obtained above it turns out to be enough to predict the fate of the mode (5.11) (which is growing in superinflationary Universe) after transition to Friedmann era.

As we have seen before the nondecaying mode of the gravitational potential in an expanding Universe after the change in the equation of state is redistributed in comparable proportions between nondecaying and decaying modes. If it would be also the case for the decaying mode the situation would be quite unfavorable for the superinflationary models. However substituting (5.11) in (4.5) we immediately find that

$$
S^{+}=S^{-}
$$

to leading order in $(k \eta)$-expansion. A more careful analysis shows that the nondecaying mode will be generated only at the next order in $(k \eta)$, that is $D^{+} \simeq(k \eta)^{2} S^{-}$. Thus we see that the growing mode of scalar perturbations in superinflationary Universe will be practically entirely converted in the decaying mode in Friedmann Universe. This result is general and doesn't depend on the concrete model for transition from superinflationary to Friedmann era. The only restriction is that the transition happens on a hypersurface of constant energy. 


\section{DISCUSSION}

We derived the matching conditions for cosmological perturbations during a sharp change in the equation of state in gauge invariant form and in different gauges (including the synchronous one) assuming that the transition from one stage to the other happens on the hypersurface of constant energy. This last restriction is quite natural and it is dictated by the physics of the transition. Using it we confirmed and clarified the standard result concerning the ratio of density perturbation to gravity waves predicted by inflationary models.

We showed that the matching conditions used in [1] do not satisfy this requirement and therefore lead to results for the spectrum of density perturbations at odds with the standard results.

The results obtained go beyond the model usually considered and can be applied also study to the fate of cosmological perturbations in models where not all of the details of the physics of the transition are specified. The only important piece of physics which we used is that the transition happens on a hypersurface of constant energy. The results were applied to follow the fate of the growing mode in superinflationary cosmology .

A final remark which we would like to make concerns the "best" gauge for cosmological perturbations. As far as we are aware the question about "what is the best" gauge or the advantage of the gauge invariant formalism is still under debate in the literature. Our (subjective) point of view is quite simple. There are of course no arguments of principle which would permit us to decide: all gauges, and even the gauge invariant formalism are on the same footing; one can work in any gauge. We hope however that in this paper we clearly demonstrated that for practical purposes the gauge invariant formalism (or equivalently the longitudinal gauge) is by far the most convenient one for treating cosmological perturbations and is an insurance against mistakes. 


\section{ACKNOWLEDGMENTS}

We thank L.P. Grishchuk for raising our interest in this problem and comments and C. Gundlach, D. Langlois, J. Martin, D. Polarski and C. Schmid for discussions. V.F. Mukhanov would like to thank the Département d'Astrophysique Relativiste et de Cosmologie for hospitality and the CNRS for financial support. 


\section{REFERENCES}

* On leave of absence from Institute for Nuclear Research, Moscow 117312.

[1] L.P. Grishchuk, Phys. Rev. D 50, 7154 (1994);

[2] V.F. Mukhanov, H.A. Feldman, R.H. Brandenberger, Phys. Rep. 215, 203-333 (1992).

[3] A. Guth, Phys. Rev. D23, 347 (1981); A. Linde, Phys. Lett. 108B, 389 (1982); A. Albrecht, P.J. Steinhardt, Phys. Rev. Lett. 48, 122 (1982); A. Linde, Phys. Lett. 129B, 177 (1983).

[4] V. Mukhanov and G. Chibisov, JETP Lett. 33, 532 (1981); A. Guth and S.Y.Pi, Phys. Rev. Lett. 49, 1110 (1982); A. Starobinski, Phys. Lett., B117, 175 (1982); S. Hawking, Phys. Lett. B115, 295 (1982).

[5] J.M. Bardeen, P.J. Steinhardt, M.S. Turner, Phys. Rev. D28, 679 (1983).

[6] V. Mukhanov, Pis'ma ZETP 41, 402 (1985); Mukhanov, Sov. Phys. JETP 67, 1297 (1988).

[7] N. Deruelle, C. Gundlach, D. Polarski, Class. and Quantum Grav. 91511 (1992).

[8] U. Gerlach and U. Sengupta, Phys. Rev. D18, 1789 (1978); J.M. Bardeen, Phys. Rev. D22, $1882(1980)$.

[9] D. Lyth, Phys. Rev. D31, 1792 (1985); R. Brandenberger in "Physics of the Early Universe", J.A. Peacock et al., SU-SSP publications 1990.

[10] N. Deruelle, C. Gundlach, D. Polarski, Class. and Quantum Grav. 9, 137 (1992).

[11] L.P. Grishchuk, in Proc. of the 3rd Course of the International School of Astrophysics, Erice, Italy, 1994; Report WUGRAV-94-11.

[12] A. Lichnerowicz, "Théories relativistes de la gravitation et de l'électromagnétisme", Masson (Paris) 1955. 
[13] G. Veneziano, "String, Cosmology,... and a Particle" talk given at PASCOS'94, preprint CERN-TH.7502/94 (November 94); M. Gasperini and G. Veneziano, Phys. Rev. D50, 2519 (1994).

[14] R. Brustein, M. Gasperini, M. Giovannini, V.F. Mukhanov, G. Veneziano, "Metric Perturbations in Dilaton-Driven Inflation", Preprint CERN-TH.7544/94 (1994) to appear in Phys. Rev. D.

[15] R. Brustein and G. Veneziano, Phys. Lett. B329, 429 (1994). 